\title{
Optimization Framework for Short-Term Control of Energy Storage Systems
}

\author{
Gustavo Aragón, Erdem Gümrükcü, José Ángel Carvajal Soto \\ Fraunhofer FIT \\ Sankt Augustin, Germany \\ \{gustavo.aragon, erdem.guemruekcue, jose.angel.carvajal.soto\}@fit.fraunhofer.de
}

\begin{abstract}
Short-term control of energy storage systems (ESS) aims to find the optimal control action for the next time step in a demand management system. Several optimization models and solution strategies are presented in literature for accomplishing this task. However, there is no framework available, which enables prototyping and flexible definition of optimization problems according to changing conditions and constellation of components in real time applications and that is deployable in different embedded systems. The present work analyses the requirements imposed by the EU project Storage4Grid (S4G) and uses them as a basis for the design of an optimization framework to combine data from various sources and offer a flexible optimization-setting environment. The architecture includes modules for management and signal processing of sensor data, linking of predictive algorithms to deliver inputs to the optimization model, optimization modeling, linking of a solver, an optimization controller and a post-processer module for formatting the results or creating events. The framework is tested on three scenarios of a deterministic optimization problem and its output interface was linked to an open source power flow simulator OpenDSS to validate the results.
\end{abstract}

Index Terms-Optimization Framework, deterministic optimization, stochastic optimization, predictive algorithms

\section{INTRODUCTION}

Smart Grids is a broad term used to identify the integration of secure, two-way communications and information technology into electrical power grids to achieve a secure, clean and efficient power supply system [1]. Smart Grids have to deal with increasing rates of decentralized and renewable generation [2] and the corresponding challenges. The volatile character of renewable generation [3] and stochastic nature of user behavior increase the control dynamics and could lead to instabilities [4]. Therefore, storage systems are crucial for the development of Smart Grids [5]. They set the ground for an efficient utilization of renewable energy sources (RES), not only supporting demand requirements, but also increasing prosumer's resilience while contributing to grid stability.

Numerous optimization models and algorithms for ESS management have been developed during the last years. For example in [6] the authors present a deterministic linear programming (LP) approach to optimize the energy storage dispatch schedule for peak shaving purpose in a grid-connected, combined photovoltaic-battery storage system. Another LP approach that optimizes total cost of operation of a stand-alone photovoltaic-diesel generator-storage system is presented in [7]. In [8], a mixed-integer nonlinear problem (MINLP) that aims at maximization of the consumption from locally generated power at a grid-connected house is covered. However, these approaches are proprietary solutions, which not offer flexibility to integrate new grid components or to adapt to dynamic scenarios.

In this work, we address the conflict between the dynamic scenario setting of household devices and controllable flexibilities due to new technologies and IT development, and the static definition of optimization problems. We propose a flexible optimization framework, which enables the integration of heterogeneous sensor data and dynamic optimization definition based on the changed conditions and constellation of components. In [9] and [10], the authors present a framework for canonical modeling of stochastic optimization problems in energy. This framework is a guidance to formulate and analyze stochastic optimization problems but is not deployable. Other frameworks are application-dependent e.g. the optimal development of one-way car-sharing system [11], or the cooperative management of batteries in a household equipped with photovoltaics (PV) regarding the individual load profile [12]. Moreover, tools for optimization modeling are available, e.g. Pyomo for python or OptimJ in Java, as well as solvers, e.g. Glpk, Bonmin, etc. These frameworks and solvers simplify the modeling of the optimization problems and the implementation of algorithms. However, they do not manage sensor data automatically, prepare it as input for the optimization models, and process the output of them for controlling other systems.

\section{BACKGROUND}

Optimization strategies and solutions for Smart Grids are rarely developed in consideration of future developments of active components and in Information and Communication Technology (ICT) potential. Therefore, a flexible tool to integrate various kinds and number of components and data from different sources provides a solution to this problem. The motivation for such a flexible optimization framework, the currently developed scenarios and the deduced requirements for the framework are presented in the following.

\section{A. Motivation}

Due to the high rate of innovation and development of ICT and to the rapidly evolving concept for Internet of Things (IoT), the number of active components in Smart Grids is growing 
continuously. This leads to new challenges regarding the feasibility of developed solutions for Smart Grids, such as energy management and optimization, and adds a restriction to their applicability. According to the predominantly static characteristics of conventional power systems, optimization strategies are tested in scenarios with conditions similar to the current conditions, or in somewhat more futuristic scenarios with notoriously increased RES penetration and high share of electric vehicles (EVs) in the grid. However, the fewest optimization strategies are tested for scenarios considering potential ICT development or further merge of Smart Grids with IoT. A factor here fore is the high level of uncertainty regarding potential, speed and direction of future developments, but also the high complexity, which is introduced by nested testing of a number of potential condition modification in future. Therefore, formerly modeled optimization problems and testing results can only be mapped to exactly the same scenarios, with the same conditions and combinations of components.

A further factor, which distinguishes the development of Smart Grids from the conventional power system, is the interdisciplinary approach involving a variety of experts with different background. The wide range goes from energy supply employees and technicians through ICT engineers up to mathematicians, physicists, etc. According to the different background and way of use of an optimization strategy, the requirements vary widely. Therefore, a scalable solution is required, to enable its use at different expertise levels.

In order to tackle these challenges, a flexible solution enabling the dynamic introduction of new components and modifying the outline of an optimization strategy would be a step in the right direction. In this work, we introduce an Optimization FrameWork (OFW), which is designed to integrate data from arbitrary sensors and enable flexible definition and application of optimization strategies.

\section{B. Scenarios}

OFW is developed within the EU project $\mathrm{S} 4 \mathrm{G}$, which is dealing with the usage of storage technologies by modeling, planning, integrating operating and evaluating distributed ESS. S4G considers three different visions of the collaborative use of ESS and EV paired with an optimized energy flow: A) Advanced Cooperative Storage Systems, B) Cooperative EV Charging, and C) Storage Coordination.

The first vision depicts a local hybrid AC/DC network environment formed by self-resilient prosumers and consumers. The goal is to minimize the energy import from the grid by balancing demand and generation in a grid segment equipped with ESS and PVs. The depicted vision is tested and developed in a laboratory setting (MicroDERLab facilities) in Bucharest, Romania.

The second vision highlights the role of PVs and EV charging stations in a storage environment for both residential and commercial usage. The commercial use case focuses on monitoring and controlling the charging of multiple EVs in a charging station featuring both slow and fast charging points. The residential use case focuses on the management of ESS and the controlling of EV charging strategies with the presence of
PV. It is deployed in Bolzano, Italy, in a single-family house, as well as a company's EV fleet park.

The third vision investigates the benefits of distributed and grid-connected storage in respect to increasing RES penetration in the existing grid and improving the end-user efficiency and resilience as a stand-alone entity. It is tested on the Island of Fur, Denmark, on five houses with PV and storage.

\section{Requirements/Blocks}

The situation described in the second vision demonstrates the need for control algorithms being able to coordinate efficiently ESS, PV generation, charging stations and the energy exchange with the grid connection point, taking into account local restrictions and priority settings.

The energy flow coordination analyzed from the point of S4G derives several requirements listed in the following:

Input information requirements: smart meters collect data about energy consumption or generation from the different electrical nodes. The smart meters used in S4G project communicate consumption data every minute through a RS232 interface managing "Device Language Message Specification" (dlms) communication protocol. This requires a central entity reading the information from the smart meters regardless of their communication protocol. This information has to be preprocessed and formatted for serving as an input for OFW.

Users: In S4G, OFW has to be deployed by electrical engineers and computer scientists working with signal processing and configuration and programming of embedded systems. These experts' knowledge in the field of deterministic and stochastic optimization, and predictive algorithms is limited. Therefore, OFW has to be designed as a tool that they can test and deploy by themselves while fully exploiting the power of the optimization algorithms. It should support their work with prototyping of demand management systems in real applications.

Output: solutions of the optimization problem have to be translated into understandable messages for the actors in the system such as ESS. In this case, software adapters are needed to translate results from the optimization into control messages and insert them in the correct communication protocol.

Operating platform: all residential premises included in the project use cases were equipped with raspberry pi 3 . Raspberry pi 3 has Raspbian as operating system, which is based on Debian (Linux distribution). This naturally limits the development of OFW in regard of computing power and operation system.

Predictive algorithms: optimization is only useful if parameter predictions for a defined horizon are taken into account. In fact, the ESS control in S4G can only be optimized if the future behavior of parameters are known or predicted. In $\mathrm{S} 4 \mathrm{G}$, an instant optimization problem is not applicable because there are several stochastic variables changing the system randomly. One example is the energy consumption behavior, which varies significantly over the year.

Optimization workflow: the control of the ESS has to be updated every 10 minutes. This implies that the framework has to be able to collect the information from the smart meters, 
predict the necessary information and calculate the optimization problem in this period.

Restrictions and prioritization: depending on the country of deployment, legal issues and regulations can vary significantly, e.g. allowance and limitations to feed energy into the grid. Besides, following a user-centered approach, needs and rights of the customer regarding the energy management in their property play a central role in the project. Preferences can range from a more environment-oriented energy flow optimization to financial gains for selling energy to the distribution system operator (DSO) and other actors.

Deployment: the system is deployed in a S4G's test site in Bolzano, Italy, which features a single-family house in a rural village with a PV and a residential charging station, as well as a company's EV fleet park with seven charging stations. Moreover, five residential houses in the island of Fur, Denmark will be also equipped with this system. The houses have photovoltaic and ESS.

\section{FRAMEWORK}

\section{A. Structure}

The first version of the OFW architecture was developed based on the requirements imposed by S4G project (see Fig. 1).

The sensor connector enables the connection of various and diverse sensors to the framework. In the case of S4G, SMX (Smart Meter Extension), a software package developed for NobelGrid EU-project, will be used [13]. SMX is dedicated to data collection from smart meters like those used in S4G. Another possibility, which has been evaluated by our team, is the sensor platform (SPF) [14]. This framework allows the connection of either wireless or cable connected sensors dynamically. The sensor connector module also allows the use of cloud services to gather specific forecast information. This information can be used instead of machine learning algorithms for the generation of a desired prediction. In the case of PV generation, this connector queries a weather forecast service for temperature and irradiation forecasts. This information inputs are used consequently by the data management and processing module to calculate a physical model.

The input data mapping module allows OFW to map the input information to the used channel and the corresponding context. In the case of S4G, smart meters constitute the sensors and their information represent a variable defining the system. For example, OFW reads the data from a smart meter from a defined Message Queue Telemetry Transport (MQTT) topic, which represents the power of ESS.

The data management and processing module manages the input data from sensors, pre-process it, links an implemented algorithm through the model loader, evaluates and executes a post-processing of the results. In fact, the output of this module are the predictions that serve as inputs for the optimization model. The time space for the predictions is restricted by the maximal optimization period as stated in the requirements. OFW uses the complex event processing and machine learning framework (CEML) in charge of preprocessing, learning, evaluating and deploying data from machine learning algorithms

[15]. CEML connects to algorithm implementations using the model loader module. This module loads models using either existing implementations or by processing a specification of the model. Models of the types, physical or machine learning are supported. Finally, all the models lifecycle are orchestrated by the data management and processing module.

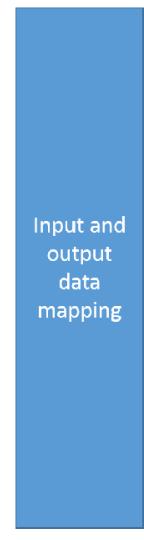

Input and output mapping

(1)

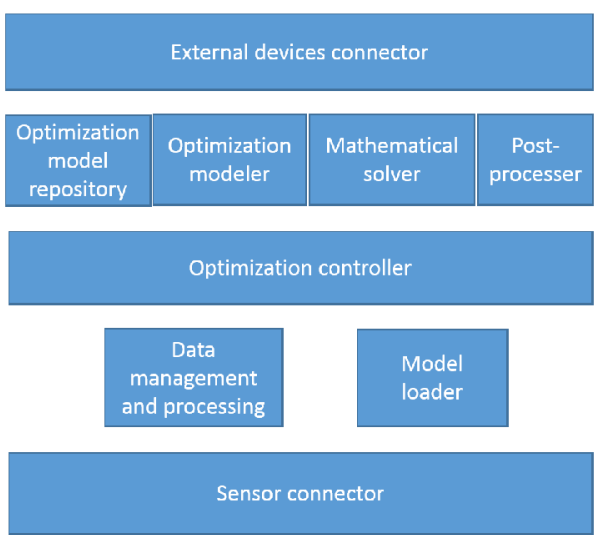

Figure 1: Architecture of OFW

The core part of OFW is the optimization controller in charge of the orchestration of output data from the data management and processing module, of incorporating the correct optimization model into the system and the consequent linking of a mathematical solver. The optimization controller manages the frequency of the output events and errors arising from the optimization process. This module uses the restriction mapping module for generating new constraints or for choosing one optimization model from the Optimization Model Repository. These repository stores optimization model definitions already tested and evaluated in systems presenting similar scenarios.

The optimization modeler applies optimization-modelling tools to define mathematically the optimization problem and to link a mathematical solver or algorithm in charge of the calculation of the modeled problem. In fact, if a set of data is present, a previous analysis of the behavior of certain optimization models can be easily achieved. This capability simplify the design of new optimization models by the users. Because of the use of open source optimization modelling tools, OFW supports a widely range of optimization problem types starting from linear programing to more complex stochastic programming problems. Thus, it enables the framework to be used and tested in different application domains such as energy, smart cities, smart factories, etc.

The post-processer prepares the results obtained from the mathematical solver to be transmitted to the actuator device. This module generates and manages event-based messages, which will be exchanged among distributed devices, e.g. in a more complex cooperative energy management scenario.

Through the external device connector the output of the optimization can be obtained through a RESTful API or subscribed if using MQTT services. 


\section{B. Implementation}

OFW is implemented in Python and runs into a Docker container. All dependencies are specified by the Docker image, in order to facilitate deployment. Therefore, to deploy OFW only few configuration steps are needed.

\section{DEMONSTRATION}

An application example of OFW for optimal control of ESS in a residential place equipped with a PV and ESS is presented and discussed (Fig. 2). In the following, the local energy targets and the corresponding optimization models are introduced, as well as a testing scenario and the results.

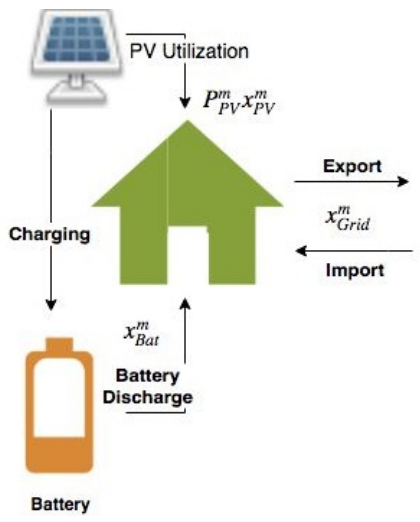

Figure 2: Residential scenario in Bolzano, Italy

\section{A. Optimization models}

In this work, we assume ideal accuracy of weather, demand and electricity price forecast. Therefore, the problem is modeled as a static and deterministic optimization problem having parameters like: maximum power output of PV panel for given weather prediction $P_{p v}^{m}$, power demand of the house $P_{d e m}^{m}$, and electricity price $C_{\text {price }}^{m}$, where $\mathrm{m}$ is the time step. Control variables in this problem are continuous: utilization rate of solar PV potential $x_{p v}^{m}$, which yields the electrical power output at time step $\mathrm{m}$ when multiplied with $P_{P V}^{m}$, power exchange with ESS $x_{B a t}^{m}$, and the power exchange with the grid $x_{G r i d}^{m}$. Following constraint ensures that electrical demand is met completely. Note that $x_{B a t}^{m}$ and $x_{\text {Grid }}^{m}$ terms can take both positive and negative values. The sign is determined based on their impact to the load. Therefore, battery discharge and import are positive, charge and export negative.

$$
P_{d e m}^{m}=P_{p v}^{m} x_{p v}^{m}+x_{b a t}^{m}+x_{G r i d}^{m}
$$

The state of charge (SoC) of the battery is a state variable in this model, which is modeled as a function of the previous SoC and the power exchange with the battery at the previous time step. Charging/discharging processes are assumed to be $100 \%$ efficient. Inserting $\frac{\Delta T}{3600}$ term, the power exchange with the battery $(\mathrm{kW})$ is translated into energy $(\mathrm{kWh}) . E_{\text {bat }}$ is the battery's energy storage capacity:

$$
x_{S O C}^{m+1}=x_{S O C}^{m}+x_{b a t}^{m} \frac{\Delta T}{3600} \frac{1}{E_{\text {bat }}}
$$

The linear constraints and continuous variables were applied to model three different objectives for the optimization problem i.e. minimization of power import/export, maximal utilization of renewable potential, and electricity bill minimization.

$$
\begin{array}{ll}
\text { minimize } & \sum_{m=1}^{M}\left(x_{\text {Grid }}^{m}\right)^{2} \\
\text { maximize } & \sum_{m=1}^{M} P_{p v}^{m} x_{p v}^{m} \\
\text { minimize } & \sum_{m=1}^{M} C_{\text {price }}^{m} x_{\text {grid }}^{m}
\end{array}
$$

In Eq. 3 a house with minimum grid exchange target should penalize the export too. In order not to incentivize negative $x_{G r i d}^{m}$ values, the grid exchange minimization objective was modelled as a quadratic function. Here $\mathrm{M}$ stands for the optimization horizon and corresponds to the number of time steps for the optimization. Eq. 4 represents the PV utilization as a linear maximization problem. Eq. 5 models a bill minimization objective, where a single price signal for the house is assumed, so that export-import cases are not subject to separate tariffing and that the objective is a linear function.

Single price tariff for export-import cases is a very rare approach in energy markets. Many countries adopted feed-in tariffs to price the locally generated power's export to the grid. However, integration of separate price tariffs for import $C_{\text {price,imp }}^{m}$ and export $C_{\text {price,exp }}^{m}$ into the objective function necessitates new variables for export-import powers $x_{g r i d, e x p}^{m}$ and $x_{\text {grid,imp }}^{m}$. Furthermore, in order to avoid simultaneous export-import, two binary variables $y_{\text {grid,exp }}^{m}$ and $y_{\text {grid,imp }}^{m}$ are needed.

$$
\begin{gathered}
x_{\text {grid }}^{m}=y_{\text {grid,imp }}^{m} x_{\text {grid,imp }}^{m}-y_{\text {grid,exp }}^{m} x_{\text {grid,exp }}^{m} \\
y_{\text {grid,exp }}^{m}+y_{\text {grid,imp }}^{m} \leq 1
\end{gathered}
$$

Eq. 6 is a nonlinear constraint that represents the power exchange as the summation of the export and import power. Eq. 7 ensures that only one of them can take place at a time $m$. Then, Eq. 5 transforms into Eq. 8, in which only one term will be nonzero at each time step. This transformation, however, changes the problem class into a non-linear mixed integer problem.

$$
\text { minimize } \quad \sum_{m=1}^{M} C_{, i m p}^{m} y_{i m p}^{m} x_{, i m p}^{m}-C_{\text {exp }}^{m} y_{\text {exp }}^{m} x_{\text {exp }}^{m}
$$

\section{B. Scenario}

To test the optimization approach, a real radial distribution network from Bolzano is simulated (see Fig. 3). The house at node 121117 is analyzed for controlling its ESS, based on the optimization models explained in IV.A. The house has a PV panel with $7.6 \mathrm{~kW}$ nominal power under standard test conditions 
and a Fronius ESS system with $9.6 \mathrm{kWh}$ energy capacity. The battery charge and discharge powers are restricted to maximal power of $6.4 \mathrm{~kW}$ available from the inverter. Fig. 4 shows the PV, demand and price forecasts for this node.

A first version of OFW was used for testing the optimization problem. The open source optimization-modelling tool for python environment Pyomo served as Optimization modeler module. The linear optimization problem was solved with different open-source mathematical solvers such as Glpk, Ipopt and Bonmin in order to diversify the problem classes that the framework is capable of solving from Linear to Mixed-Integer Linear, Non-Linear, and Non-Linear Problems. The Optimization controller managed the input data and mapped it into the correct channels. The post-processer was used for wrapping the information to make it suitable to use in OpenDSS. In this version, a unique point optimization solution was established for analyzing processing time and platform independence of the framework.

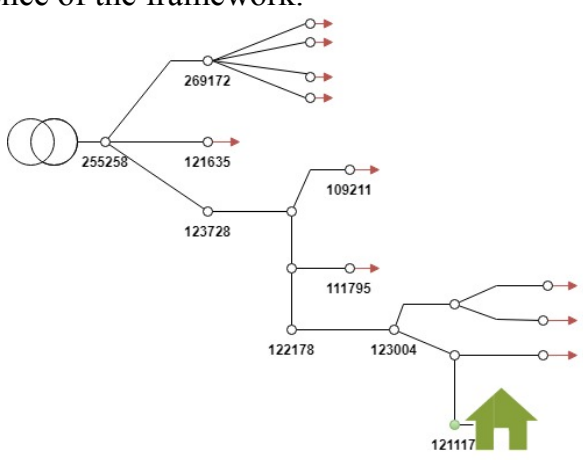

Figure 3: Distribution network in Bolzano, Italy

\section{Results}

As a primary performance indicator, the processing time for accomplishing a calculation of the optimization problem is used. Since the time resolution is set as one minute for a horizon of one day, the optimization horizon is 1440 time steps. There are 1440 values of each control variables $\left(x_{\text {Grid }}^{m}, x_{B a t}^{m}, x_{p v}^{m}\right)$ and 1441 state variables $x_{\text {SOC }}^{m}$ (including final time step). Demand meeting (1) and battery storage (2) constraints must be met at each point of time. Therefore, there are totally 2880 constraints in our optimization problem. The computations were done using a computer with Intel(R) Core(TM) i7-4800MQ CPU 2.70 GHz. Table 1 shows the processing time in seconds for each optimization model and compares different solvers. Note that Glpk is unable to handle quadratic objective functions, therefore it is not used for solving the 'Grid Exchange Minimization' problem.

Moreover, local performance indicators are defined to compare the optimization models. The indicators are divided into imported/exported energy, PV utilization, electricity bill and battery lifetime. The values were obtained from the power flow simulation with OpenDSS by applying the optimal ESS schedules obtained from OFW. The impact on the battery lifetime is quantified by using a micro-cycle counting method known as Rainflow counting [16]. This method evaluates the stress exerted on the battery. Table 2 shows the results.
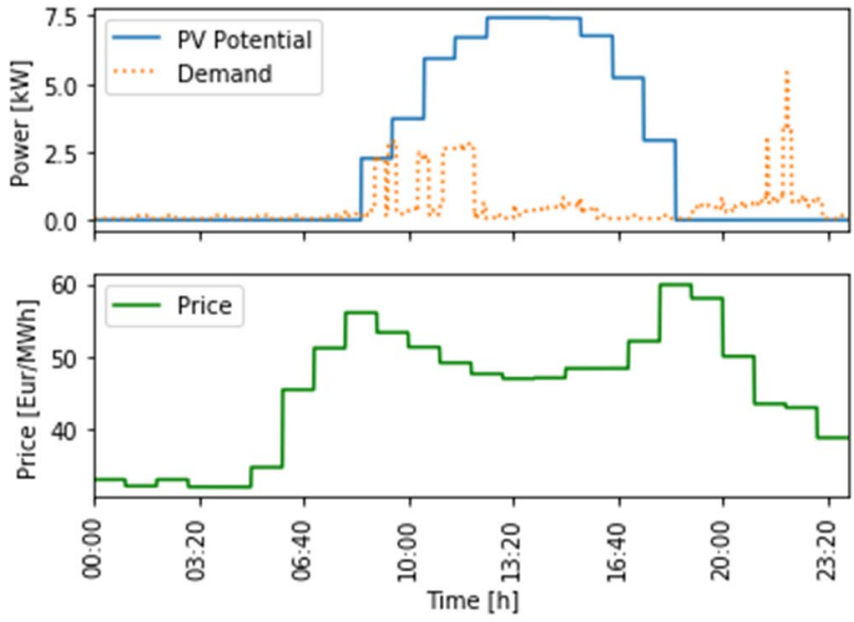

Figure 4: PV generation, load and price forecasts used in the optimization

TABLE 1: PROCESSING TIME [S] OF DIFFERENT SOLVERS

\begin{tabular}{|l|l|l|l|}
\hline \multicolumn{1}{|c|}{ SOLVER } & \multicolumn{1}{|c|}{ Glpk } & \multicolumn{1}{c|}{ Ipopt } & \multicolumn{1}{c|}{ Bonmin } \\
\hline Solver capability & LP, MILP & $\begin{array}{l}\text { LP, } \\
\text { QP, NLP }\end{array}$ & $\begin{array}{l}\text { LP, MILP, QP, } \\
\text { NLP, MINLP }\end{array}$ \\
\hline $\begin{array}{l}\text { Grid Exchange } \\
\text { Minimization }\end{array}$ & ------ & 0.193 & 0.190 \\
\hline $\begin{array}{l}\text { PV Utilization } \\
\text { Maximization }\end{array}$ & 0.059 & 0.159 & 0.145 \\
\hline Power Bill Minimization & 0.106 & 0.274 & 0.251 \\
\hline
\end{tabular}

TABLE 2: LOCAL PERFORMANCE INDICATORS

\begin{tabular}{|l|c|c|c|c|c|}
\hline $\begin{array}{c}\text { Optimization } \\
\text { Objective }\end{array}$ & Import & Export & $\begin{array}{c}\text { PV } \\
\text { Utilization } \\
\text { \% }\end{array}$ & $\begin{array}{c}\text { Energy } \\
\text { Bill } \\
\text { EUR }\end{array}$ & $\begin{array}{c}\text { Battery } \\
\text { Degrad. } \\
\text { \%/year }\end{array}$ \\
\hline $\begin{array}{l}\text { Grid Exch- } \\
\text { Minimization }\end{array}$ & 0.00 & 0.00 & 20.2 & 0.00 & 5.9 \\
\hline $\begin{array}{l}\text { PV Util. } \\
\text { Maximization }\end{array}$ & 5.82 & 48.84 & 100.0 & -1.74 & 0 \\
\hline $\begin{array}{l}\text { Power Bill } \\
\text { Minimization }\end{array}$ & 24.5 & 70.80 & 100.0 & -2.19 & 71.8 \\
\hline
\end{tabular}

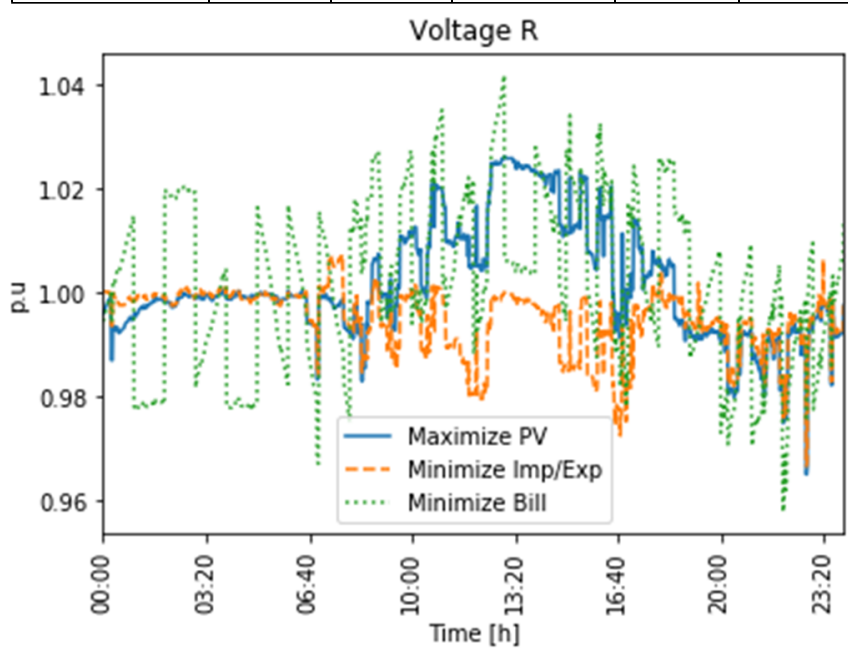

Figure 5: Voltage profile (p.u.) on the bus

TABLE 3: NL COMPUTATION PERFORMANCE OF BONMIN

\begin{tabular}{|c|l|l|l|l|l|l|}
\hline$\Delta \boldsymbol{T}[\mathbf{m i n}]$ & 60 & 30 & 20 & 15 & 10 & 5 \\
\hline Time [sec] & 1.23 & 5.13 & 5.35 & 8.83 & 9.55 & 53.59 \\
\hline
\end{tabular}


In addition to the local performance criteria, ESS control strategies' impact on the voltage profile at the bus are investigated. Fig. 5 shows the voltage profile normalized according to the nominal voltage of the bus.

Finally, computation performance of Bonmin solver in solving NL problems with different time resolutions is investigated. Table 3 shows the computation times of the nonlinear mixed-integer problem (including constraints Eq. 6-7) with the grid exchange minimization objective for different time resolutions.

\section{CONCLUSIONS AND OUTLOOK}

The optimization framework presented in this work was evaluated using the implementation flexibility of different optimization models, the linking of different solvers, as well as the computation time as criteria. In this context, three different linear deterministic optimization models corresponding to minimization of power import/export, maximal utilization of renewable potential, and electricity bill minimization were tested. The models were solved linking three different noncommercial mathematical solvers, such as Glpk, Ipopt and Bonmin, while the computation time was stored. The computation time remained in the range of hundreds of milliseconds with all optimization models and solvers and below one minute with the NLMIP. Due to the 10-minute control loop frequency set in S4G-Project, OFW can be used as a short-term continuous control of ESS in residential places.

OFW was also linked to a power flow simulator to analyze the optimized ESS schedules. For the comparison of the optimization models, a list of local performance indicators is suggested, such as the import/export energy to the grid, the percentage of PV utilization, the energy bill in euros and the battery degradation per year. Furthermore, the global impact of the optimization models in the grid was evaluated by monitoring the voltage profile on the connection point of the electrical node to the bus. A real grid segment in Bolzano, Italy was used in the simulation. As expected, the best voltage performance was documented when storage is operated according to the grid exchange minimization criteria.

Further research will focus on the extension of OFW to stochastic optimization problems, as well as on the implementation of different predictive algorithms and the integration of electric vehicles' charging approaches. The introduction of an optimal cooperative control of flexibilities is also envisioned in OFW.

\section{ACKNOWLEDGMENT}

This project has received funding from the European Union's Horizon 2020 research and innovation program under grant agreement No 731155. The sole responsibility of this publication lies with the author. The European Union is not responsible for any use that may be made of the information contained therein.

\section{REFERENCES}

[1] IEEE Std 2030-2011, IEEE Guide for Smart Grid Interoperability of Energy Technology and Information Technology Operation with the Electric Power System (EPS), End-Use Applications, and Loads, no. September. 2011.

[2] L. Barelli, G. Bidini, and F. Bonucci, "A micro-grid operation analysis for cost-effective battery energy storage and RES plants integration," Energy, vol. 113, pp. 831-844, 2016.

[3] I. Stoyanova, M. Marin, and A. Monti, "Characterization of load profile deviations for residential buildings," 2013 4th IEEE/PES Innov. Smart Grid Technol. Eur. ISGT Eur. 2013, pp. 1-5, 2013.

[4] W. F. Pickard, A. Q. Shen, and N. J. Hansing, "Parking the power: Strategies and physical limitations for bulk energy storage in supply-demand matching on a grid whose input power is provided by intermittent sources," Renew. Sustain. Energy Rev., vol. 13, no. 8, pp. 1934-1945, 2009.

[5] X. Luo, J. Wang, M. Dooner, and J. Clarke, "Overview of current development in electrical energy storage technologies and the application potential in power system operation," Appl. Energy, vol. 137, pp. 511-536, 2015.

[6] A. Nottrott, J. Kleissl, and B. Washom, "Storage dispatch optimization for grid-connected combined photovoltaic-battery storage systems," IEEE Power Energy Soc. Gen. Meet., pp. 1-7, 2012.

[7] C. Bordin, H. O. Anuta, A. Crossland, I. L. Gutierrez, C. J. Dent, and D. Vigo, "A linear programming approach for battery degradation analysis and optimization in offgrid power systems with solar energy integration," Renew. Energy, vol. 101, pp. 417430, 2017.

[8] M. Castillo-Cagigal et al., "PV self-consumption optimization with storage and Active DSM for the residential sector," Sol. Energy, vol. 85, no. 9, pp. 2338-2348, 2011.

[9] W. B. Powell and S. Meisel, "Tutorial on Stochastic Optimization in Energy - Part I: An Energy Storage Illustration," IEEE Trans. Power Syst., vol. 31, no. 2, pp. 1468-1475, 2016.

[10] W. B. Powell and S. Meisel, “Tutorial on Stochastic Optimization in Energy_-Part II: An Energy Storage Illustration," IEEE Trans. Power Syst., vol. 31, no. 2, pp. 1468-1475, 2016.

[11] B. Boyaci, K. G. Zografos, and N. Geroliminis, "An optimization framework for the development of efficient one-way car-sharing systems," Eur. J. Oper. Res., vol. 240, no. 3, pp. 718-733, 2015.

[12] Y. Iwafune, T. Ikegami, J. G. D. S. Fonseca, T. Oozeki, and K. Ogimoto, "Cooperative home energy management using batteries for a photovoltaic system considering the diversity of households," Energy Convers. Manag., vol. 96, pp. 322-329, 2015.

[13] "NobelGrid." [Online]. Available: http://nobelgrid.eu/. [Accessed: 16-Jul-2018].

[14] G. Aragón, R. Reiners, and Z. Kemény, "A Configurable Sensor Platform for Cloud-Based IoT Application Scenarios," in IBM Research Report: Proceedings of the 11th Advanced Summer School on Service Oriented Computing, 2017, pp. 49-52.

[15] J. Á. Carvajal Soto, M. Jentsch, D. Preuveneers, and E. IlieZudor., "CEML: Mixing and moving complex event processing and machine learning to the edge of the network for IoT applications," IoT 2016, pp. 103-110, 2016.

[16] Y. Shi, B. Xu, and B. Zhang, "Optimal Battery Control Under Cycle Aging Mechanisms,” 2017. 